\title{
Reduction of Superconducting Wave Packets in Dispersion Dynamics
}

\author{
Antony J. Bourdillon \\ UHRL, San Jose, CA, USA \\ Email: bourdillona@sbcglobal.net
}

How to cite this paper: Bourdillon, A.J. (2020) Reduction of Superconducting Wave Packets in Dispersion Dynamics. Journal of Modern Physics, 11, 365-377.

https://doi.org/10.4236/jmp.2020.113023

Received: February 13, 2020

Accepted: March 6, 2020

Published: March 9, 2020

Copyright (c) 2020 by author(s) and Scientific Research Publishing Inc. This work is licensed under the Creative Commons Attribution International License (CC BY 4.0).

http://creativecommons.org/licenses/by/4.0/ (c) (i) Open Access

\begin{abstract}
Two problems in solid state physics and superconductivity are addressed by applications of dispersion dynamics. The first is the Hall effect. The dynamics of charges that yield positive Hall coefficients in material having no mobile positive charges have always been problematic The effect requires both electric and magnetic response, but magnetic deflection is only possible in mobile charges. In high temperature superconductors, these charges must be electrons. Contrary to Newton's second law, their acceleration is reversed in crystal fields that dictate negative dispersion. This is evident in room temperature measurements, but a second problem arises in supercurrents at low temperatures. The charge dynamics in material having zero internal electric field because of zero resistivity; and zero magnetic field because of the Meissner-Ochsenfeld diamagnetism; while the supercurrents themselves have properties of zero net momentum; zero spin; and sometimes, zero charge; are so far from having been resolved that they may never have been addressed. Again, dispersion dynamics are developed to provide solutions given by reduction of the superconducting wave packet. The reduction is here physically analyzed, though it is usually treated as a quantized unobservable.
\end{abstract}

\section{Keywords}

Reduction, Wave Packet, Dispersion Dynamics, Special Relativity, Propagation, Transverse Plane, Functions of Relativistic Free Particles, Quantum Physics, Quantum Mechanics

\section{Introduction}

Dispersion dynamics grows immediately out of Special relativity by the joint introduction of Planck's law and the de Broglie hypothesis. After first order differentiation of the standard formulae $E^{2}=p^{2} c^{2}+m_{o}^{2} c^{4}$ (where $E$ represents 
energy, $p$ momentum, $c$ the speed of light, and $m_{o}$ rest mass) and the wave equivalent $\omega^{2}=k^{2} c^{2}+m_{o}^{2} c^{4} / \hbar^{2} \quad$ (where $\omega$ represents angular frequency, $k$ wave vector, and $\hbar$ the reduced Planck constant) [1]:

$$
\frac{\mathrm{d} \omega}{\mathrm{d} k} \cdot \frac{\omega}{k}=v_{g} \cdot v_{p}=c^{2}
$$

i.e. the product of phase velocity $V_{p}$ (angular frequency/wave vector) with group velocity $V_{g}$ (the first derivative $\mathrm{d} \omega / \mathrm{d} k$ ) is equal to the square of the speed of light. Alternative normalization gives $v_{g}^{\prime} \cdot v_{p}^{\prime}=1$, where $v_{g}^{\prime}=v_{g} / c$ and $v_{p}^{\prime}=v_{p} / c$. The group velocity is the speed of the particle in special relativity; the phase velocity is faster than light, and is measured either as the inverse of the group velocity or as the ratio of energy to momentum ${ }^{1}$. The phase velocity is singular in the rest frame: $v_{p} \rightarrow \infty$ when $k \rightarrow 0$. Massless particles in vacuo have $v_{p}=v_{g}$. More generally, $v_{p}=\left(1+m_{o}^{2} / k^{2}\right)^{1 / 2}$, in units $c=\hbar=1$; and $v_{g}=k /\left(k^{2}+m_{o}^{2}\right)^{1 / 2} \simeq k / \omega$ when $k \ll m_{o}$, and $m_{o} \simeq \omega$.

Previously we have explored implications for Equation (1) in many fields including wave packets [2]; antiparticles in force fields [3]; electromagnetic interactions in wave groups [4]; the stable wave packet in quantum mechanics [5]; and in uncertainty [6] that is further developed below; voids in the Hall effect [7] and excitons in high temperature superconductors; many-body-gravitational solutions for galactic rotational velocities [8] that obviate dark matter and dark energy; magnetic radius in intrinsic spin that is equal to the Compton wavelength [9], etc. These applications spring clean old cobwebs and provide many cases of improved clarity for the way forward. Now we turn our attention to the reduction of the wave packet which is implicit in all calculations that involve transitions between time-independent wave functions, and which is particularly implicit in superconductivity.

Mathematicians choose their axioms; physicists falsify them. Typically, we investigate a new problem with simplicity, expecting complexity to later resolve oversimplification. As an early example, Newton believed light is corpuscular, moving in straight lines, where the complexity of wave-particle duality, came to dominate the subsequent legacy of $19^{\text {th }}$ and $20^{\text {th }}$ Century physics. The corresponding theoretical postulate of point particles ${ }^{2}$, however convenient for limited purposes, poses anomalies for the real world of physics. For example, the electron may be regarded variously as a propagating wave in the electron microscope; or as an atomic wave orbital; or as an intrinsic magnetic current loop [9]; or having electrostatic radius. Each of these has dimensions over widely different orders of magnitude [10]. In particle physics, further dimensions occur in meson and hadron particles, and in their constituent quarks and interacting bosons.

Even at the relatively large scale of the atom, simplification is employed in ${ }^{1}$ The group and phase velocities supposedly combine to produce Dirac's “jitter" [Dirac, P.A.M., The Principles of Quantum Mechanics (1958) 4th Edition, Oxford] without purportedly offending the requirement for infinite energy in his electron particle, that he claims to have velocity $c$.

${ }^{2}$ Dirac supposed point particles apparently because the wave packet was, to him, unstable [ibid.]. 
time independent, wave functional solutions, where time is re-introduced through perturbations. The wave function then becomes a probability amplitude for locating an extreme point particle. This feature becomes more mysterious when it is reduced to reality during quantum measurement. Mystery and physics are antithetical. In particular, suppose a particle, say a photon, is emitted by an atom near point $A$ so that it may contemporaneously arrive near $B$ or $C$ with appropriate probabilities on the same wave front. Two anomalous possibilities occur: on the one hand ask, "Can the particle be absorbed at both $B$ and $C$ by breaking energy conservation and doubling energy"; or on the other, "If absorption occurs at $B$, how does information arrive at $C$-without breaking the group velocity, $v$, constraints in special relativity ( $v \leq c$, the speed of light) -in order to prevent a second absorption and to conserve energy?" A new solution for the reduction of the wave packet is available to Dispersion Dynamics [1] where the wave phase and particle group in the duality are treated specifically, having various properties that were previously ignored in standard quantum theory.

In the following discussion we suppose an objective reality in our mathematical framework that is not limited by Bohr's usage of phenomena [11]: "In contrast to his view that the notion of phenomenon irrevocably includes the specifics of the conditions of experimental observation, Einstein held that one should seek for a deeper-lying theoretical framework which permits the description of phenomena independently of these conditions"3.

Before proceeding to a treatment of reduction, it will be necessary to clarify the notion of uncertainty in all its variations through multiple dimensions. The noteworthy example in superconductivity requires prior treatment of the Hall effect. In particular, high temperature superconductors, besides being ionic, have critical temperatures $T_{c}$ that are typically an order of magnitude greater than classical, metallic, low temperature superconductors. Above respective critical temperatures $T>T_{\mathcal{o}}$ the former group displays positive Hall coefficients, $R_{H}>0$; the latter negative. The Hall voltage is generated in two stages: by a transient acceleration of electrons, across an applied magnetic field; in which magnetic deflection in the Lorentz force builds a charge potential in the steady state. The consequent field is normal to the two applied fields and produces a corresponding Hall voltage. When charge carriers are positive ions the Hall coefficient, $R_{H}>$ 0 ; typically, negative charges yield $R_{H}<0$. In high temperature superconductors, the charge carriers produce $R_{H}>0$, though the only positive charges are statio${ }^{3}$ Pais [4] wrote that "It was [Einstein's] almost solitary conviction that quantum mechanics is logically consistent, but that it is an incomplete manifestation of an underlying theory in which an underlying objective reality is possible." Actually, in his EPR paper [Einstein, A., Podolsky, B. and Rosen, N., Phys.Rev. 47 777-780 (19350] Einstein held that Bohr's theory is incomplete. The latter had claimed that all that can be known about an electron is in its wave function, particularly with regard to momentum and position. This seems an unlikely theory since Gödel's mathematical theorems on completeness and consistency in axiomatic systems [Gödel, K., Monatshefte für Mathematik und Physik 38 173-198 doi: 10.1007/BF01700692]. Popper was another realist in common with Einstein [Popper, K.R., Quantum theory and the schism in physics, Ed.Bartley W.W. III, (1982) Hutchinson]. This footnote does not contribute to the debate so much as outline background for arguments given in the text. 
nary ions. These charges are subject to negative dispersion in ionic crystal fields [1] [7]. The true motion is described in dispersion dynamics.

A second and worse anomaly is the common description of superconducting currents. These are due to Cooper pairs of electrons that co-exist, in the superconducting state, with normal electrons [12]. In the superconductor not only is $\boldsymbol{E}=0$ because of zero resistance; so also is magnetic field force $\boldsymbol{B}=0$ because of the Meissner-Ochsenfeld effect (type II superconductors are slightly more complicated); while the net momentum carried by a Cooper pair $K=0$; and likewise $\boldsymbol{S}=0$. Moreover, in the high temperature superconductor, the excitonic net electric charge $Q=0$ [1]. Yet in spite of all of these absences, a supercurrent is observed. How can this be and what are the charge dynamics? Dispersion dynamics is used to show how the wave packet is reduced to produce the external current.

\section{Uncertainty}

In mathematical quantum mechanics the wave function is a probability amplitude for finding a point particle that is bounded by limits given by the Heisenberg uncertainty principle. By contrast, classical wave mechanics contains many techniques for predicting wave behaviors that depend on particular circumstances: the image plane; or near field; or far field; whether longitudinal or transverse; whether 1-dimensional; or 2-dimensional; or spherically symmetric; or cylindrical etc. Physical uncertainty describes properties of wave groups [2] where the alternative idea of a "limit" is less specific, and less useful to experimentalists, than "expected uncertainty" [5]. This is peculiar to each of the model systems to be described. We will consider first, the direction of propagation and then the transverse direction. in which the longitudinal wave packet is directly used. Waves are bounded in various ways. Waves that are not bounded have vanishing amplitude. Bounding in either space or time increases uncertainty in wave vector or angular frequency respectively, and vice versa.

\subsection{Longitudinal}

The stable wave packet $\phi$ is defined by mean angular frequency $\bar{\omega}$ and mean wave vector $\bar{k}$, which together stabilize the packet through conservation laws in energy and momentum (Figure 1):

$$
\phi=A \cdot \exp \left(\frac{X^{2}}{2 \sigma^{2}}+X\right) \text {, with } X=i(\bar{k} x-\bar{\omega} t)
$$

where $\sigma$ is the coherence, $A$ is the wave amplitude, and $x, t$ are coordinates in space and time. We can define the uncertainty in time by the full width at height $1 / e([3]$ p. 11):

$$
\Delta t=2 \frac{\sigma}{\bar{\omega}}
$$

and uncertainty of frequency by Fourier transform: 


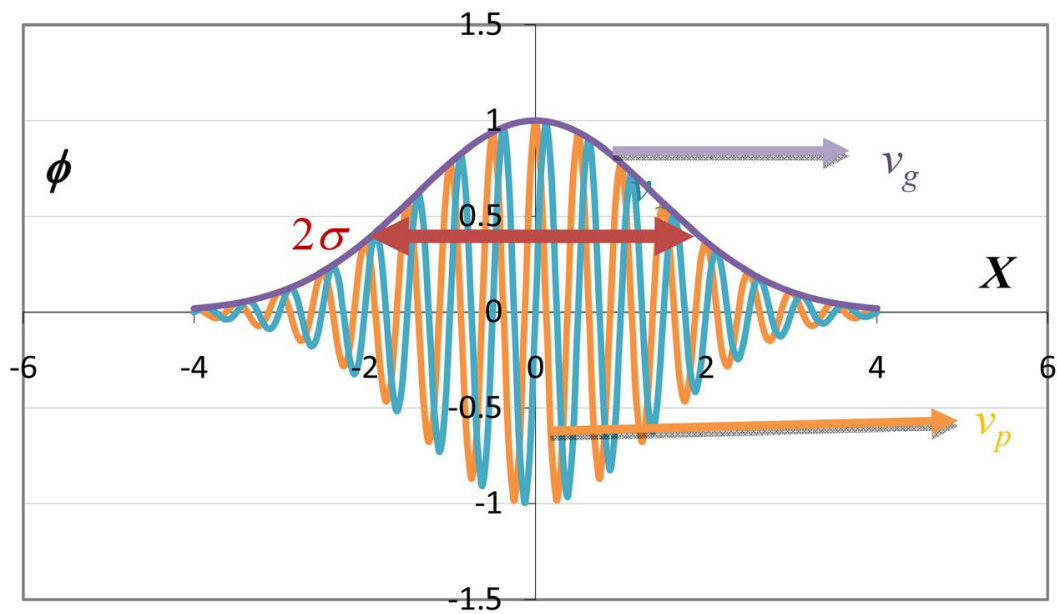

Figure 1. Stable wave packet (Equation (2)) containing envelope with group velocity $v_{g}$ (purple arrow) and real (orange) and imaginary (blue) parts of the carrier wave having phase velocity $v_{p}$ (orange arrow). The full width half maximum at $\phi=1 / e$,

$2 \sigma=\Delta t \bar{\omega} / 2=4 \bar{\omega} / \Delta \omega=\Delta x \bar{k} / 2=4 \bar{k} / \Delta k$ is shown by the red double arrow.

$$
\Delta \omega=4 \frac{\bar{\omega}}{\sigma}
$$

The product is a dual uncertainty in which $\sigma$ cancels! By substituting for $\omega$ and using Planck's law:

$$
\Delta E \cdot \Delta t=8 \hbar
$$

and by similar operations on de Broglie's hypothesis:

$$
\Delta p_{x} \cdot \Delta x=8 \hbar \text {. }
$$

Both values are more than an order of magnitude greater than Heisenberg's limit, ( $\hbar / 2$ ) (Figure 2). We shall see how they compare with uncertainty in the transverse plane. However, notice that limits are limitless, and valueless when they are too far away. In solid state theory the wave function is usually represented as indefinitely extended; but if extension is not a finite $\sigma$, the amplitude would be quantized zero.

\subsection{Transverse 1-D in the Far Field}

Next consider diffraction in the transverse plane that is caused by a horizontal slit, width $d$. The diffracted beam intensity tends to zero when the angle away from axis $\theta=\tan ^{-1}(\lambda / d)$ where $\lambda$ represents wavelength, and $\theta$ is sufficiently small. Then, using the de Broglie relationship, $p_{x}=h / \lambda$ and $\Delta p_{y}=2 p_{x} \sin (\theta)=2 h / d \quad$ ([13] p.321), where $h$ is Planck's constant.

$$
\Delta p_{y} \cdot \Delta y=2 h \text {. }
$$

This value is again greater than Heisenberg's limit, here by the factor $8 \pi$.

\subsection{Transverse 1-D in the Near Field}

It is well known that in Fresnel diffraction a narrow slit pinches a transmitted 


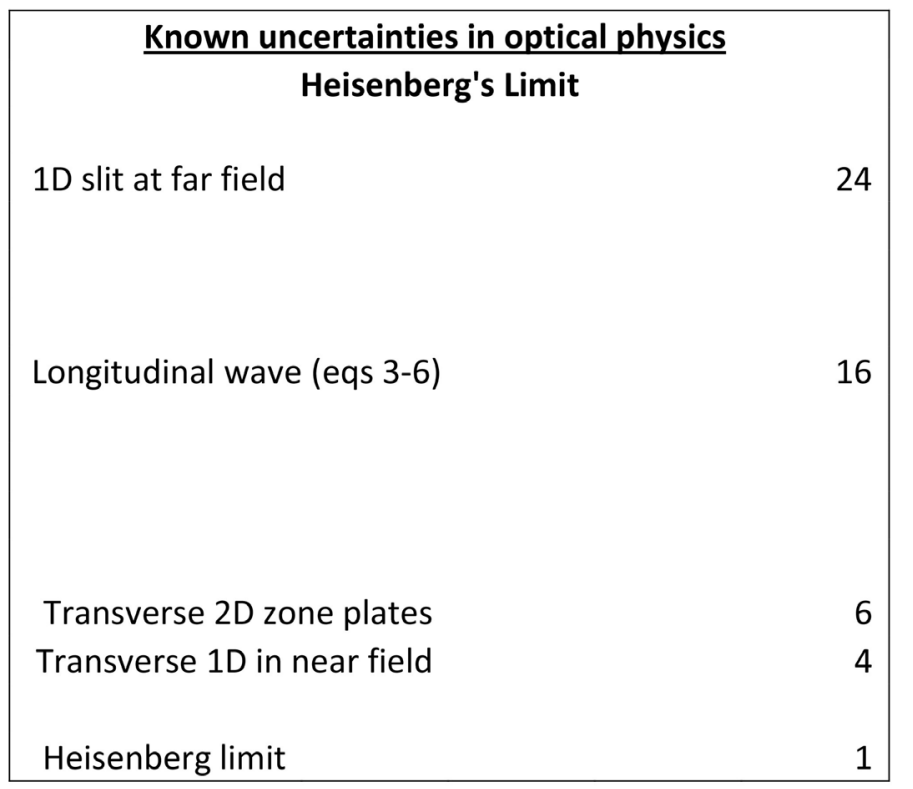

Figure 2. Uncertainties known in optical physics compared as multiples of Heisenberg's Limit.

beam in the near field, which is contrary to general effects elsewhere [13]. A zone in the wave field that has been used in X-ray lithography is called the Critical Condition [14] [15] [16] [17]. At this transverse plane, at a distance $\sim d^{2} /(3 \lambda)$ downstream from the slit, the beam width is $\sim d / 3$, From this it follows that $\Delta p_{y}=h / \lambda \cdot d / 3 \cdot 3 \lambda / d^{2}=h / d$, and:

$$
\Delta p_{y} \cdot \Delta y \sim h / 3 .
$$

The dual uncertainty is 6 times smaller than in Equation (7) and over 4 times larger than Heisenberg's limit. However, at gaps away from Critical, the contracted beam dimension varies rapidly and is illustrated elsewhere [13].

\subsection{Transverse 2-D in Zone Plates}

When a circular opaque screen of diameter $\Delta s$, is placed transverse to a parallel beam and viewed from downstream, an illuminated spot is seen on the axis. The intensity of the spot is greatly amplified when the circle is divided into optical half zones, of dimensions such that only zones of either odd or even sequence transmit. This zone plate acts like a lens [13], and aerial images show how beam profile varies with Gap $d$ [18]. A typical dual uncertainty when the number of half zones is $N_{F}=(\Delta s)^{2} /(4 d \lambda)$, is given by $\Delta p_{y} \cdot \Delta y=\Delta p_{z} \cdot \Delta z=h / 2$, i.e. 4 times smaller than Equation (7) and $2 \pi$ times greater than Heisenberg's limit. The varieties of uncertainty outlined by specific instances is needed in realistic applications of reduction in wave packets. To understand reduction, a limit of uncertainty is insufficient; only the expected value will do and this will vary with circumstances as already shown by the examples here in Section 2. The latter two cases are particularly extreme, yet are both significantly greater than Heisenberg's limit which is an inaccurate short cut. Moving forward, we must under- 
stand the electronic "hole" before applying uncertainty to superconductivity.

\section{The ELectronic "Hole" in Dispersion Dynamics}

The problem we address is this: how is a hole deflected in a magnetic field? This is supposed to occur in the Hall effect measured in a p-type semiconductor, $P$ doped $S i$ for example. The charge carrier has a positive Hall coefficient like a positive ion such as a proton or $\mathrm{Li}^{+}$ion in whatever matrix. In the two step process, the charge is first accelerated in an electric field and then deflected in a crossed magnetic field. The force $F$ on the deflection depends on the charge and is proportional to its velocity, $V_{g}$. Including the first stage electric acceleration. Instantaneously:

$$
\boldsymbol{F}=q \boldsymbol{E}+q \boldsymbol{v}_{g} \times \boldsymbol{B}
$$

Where $q$ represents the carrier charge, now presumed electronic, negative $e, E$ represents the applied Coulomb field; and $B$ the crossed magnetic intensity. Since $V_{g}$ is parallel to $E$, the magnetic part $\boldsymbol{F}_{m}$ is normal to both $\boldsymbol{E}$ and $\boldsymbol{B}$ (Figure 3 ). The velocity is produced by the electric field, and can be averaged. For the more common case of the n-type semiconductor:

$$
\boldsymbol{F}=-e \boldsymbol{E}-e \boldsymbol{v}_{g} \times \boldsymbol{B}
$$

So that the acceleration a can be written approximately:

$$
a \simeq-\frac{e \boldsymbol{E}}{m}-\frac{e \boldsymbol{E} t^{\prime}}{m} \times \boldsymbol{B} .
$$

where $t^{\prime}$ is a kind of mean time between collisions or the inverse of the number of collisions per unit time (Table 1$)^{4}$.

Since the only positive charges in $p$-type $S$, for example, are on immobile nuclei, it is obvious that the carriers cannot be ions; they must be electrons. To understand how the charges react like positive ions to produce positive Hall coefficients, we have to return to Einstein's special relativity and derive the second derivative following Equation (1):

$$
\frac{\mathrm{d}^{2} \omega}{\mathrm{d} k^{2}}=\frac{\mathrm{d} v_{g}}{\mathrm{~d} k}=\frac{\mathrm{d} v_{g}}{\mathrm{~d} p}=\left(\frac{1}{m^{\prime}}-\frac{k^{2}}{m^{\prime 3}}\right)=\frac{1}{m_{\text {eff }}}=\frac{a}{F}
$$

where $m^{\prime}$ is the relativistic mass $m_{o} /\left(1-v_{g}^{2} / c^{2}\right)^{1 / 2}$; effective mass $m_{\text {eff }}$ is as defined in the brackets; $a$ is acceleration in Newton's second law of motion corresponding to applied force $F$, such as the electric force (Equation (9)). in electricity and magnetism. Notice that a negative second derivative, or curvature (in $p$-Si, $A l$ etc.), causes negative effective mass and negative acceleration under the Coulomb force. By contrast, the magnetic force is normal to the velocity and applied magnetic intensity so that its potential is structured differently from those in electrostatics and gravity. The result is that in magnetism, $\delta \omega=0$ and the effective mass responding to the magnetic part of the electromagnetic Lorentz force is conventional (see Figure 3):

${ }^{4}$ This description has the same Hall result as previously [1] [7], but equations 10 and 11 had been misapplied to the magnetic field instead of the electric field. 
Table 1. Commonly, the "hole" is supposed to operate as a positive charge. But the Hall effect in specimens where all positive charges are immobile can only have negatively charged carriers. These are not influenced by magnetic fields. However, following dispersion dynamics, (especially columns 2 and 4 ) the mobile electrons, as illustrated in Figure 3, are influenced by applied Coulomb forces and Lorentz magnetism. These are tabulated in the double event, namely electric acceleration and magnetic deflection.

\begin{tabular}{cccccccccccc}
\hline & Charge $q$ & $D^{ \pm}$curvature & $m_{\text {eff }}$ & $F_{z}^{e l}$ & $a_{z}^{e l}=F_{z} / m$ & $s$ & $\left\langle v_{g z}\right\rangle \simeq a t^{\prime} / 2$ & $F_{x}^{m}$ & $a_{x}^{m}=F_{x} / m$ & $q \cdot a_{x}^{m} \simeq E_{x}^{\text {Hall }}$ \\
\hline $\begin{array}{r}\text { tve ion } \\
\text { Electron }\end{array}$ & $e$ & $>0$ & $m_{i}$ & $e E$ & $e E / m$ & $>0$ & $e E t^{\prime} / 2 m$ & $e B\left(e E t^{\prime} / 2 m\right)$ & $e B\left(e E t^{\prime} / m m^{2}\right)$ & $>0$ \\
$\begin{array}{c}\text { Holey } \\
\text { electron }\end{array}$ & $-e$ & $>0$ & $m$ & $-e E$ & $-e E / m$ & $<0$ & $-e E t^{\prime} / 2 m$ & $e B\left(e E t^{\prime} / 2 m\right)$ & $e B\left(e E t^{\prime} / m^{2}\right)$ & $<0$ \\
\hline
\end{tabular}

Glossary: $m_{i}$ ionic mass; $a_{z}^{e l}$ electric acceleration; $t^{\prime}$ time between collisions: expected velocity is half this time $\mathrm{x}$ acceleration; $\left\langle\boldsymbol{v}_{g z}\right\rangle$ mean z-component velocity.

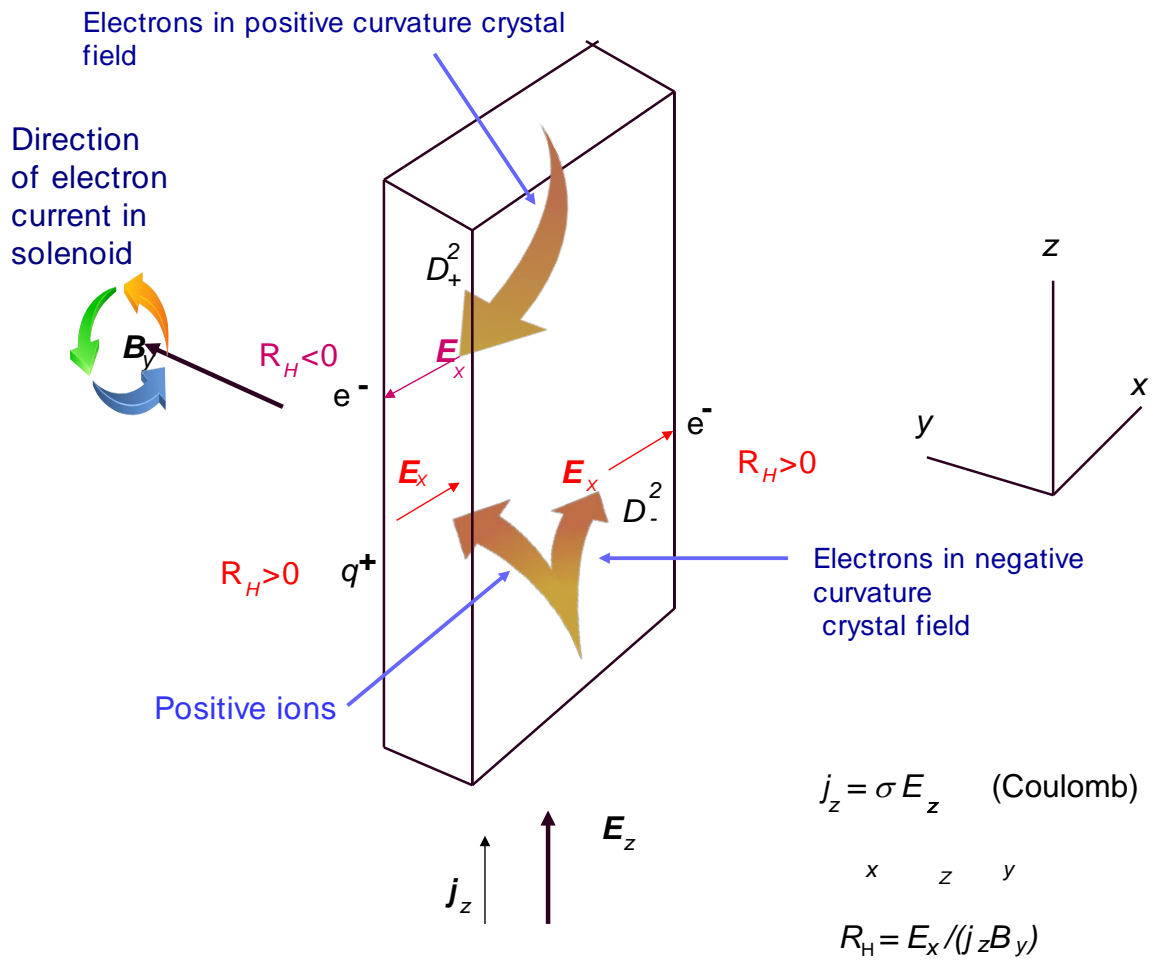

Figure 3. In the Hall effect, an applied electric field $E_{z}$ is crossed with an applied magnetic field $B_{y}$. The resulting transient current is deflected and the consequential steady state charge causes a transverse electric field $E_{X}$. Equations (9)-(12) show how $R_{H}$ is positive for mobile ions (lower left brown arrow) for p-type "holey" electrons (lower right); but more generally negative for n-type electrons (upper arrow). In dispersion dynamics, the current density $j_{z}$ of p-type electrons reverses owing to negatively dispersed crystal fields.

$$
\frac{\mathrm{d} v_{g}}{\mathrm{~d} k}=\frac{1}{m}=\frac{a_{x}}{F_{x}^{m}} .
$$

These formulae describe the motion of a single electron in an electron gas; they should be multiplied by carrier density to find measured values.

In summary:

1) The Hall effect occurs in two moments: a linear Coulomb acceleration; and 
a magnetically deflected charge. The Hall voltage results from the steady state deflection of charges.

2) As for free electrons, in typical metals (e.g. Cu) the normal dispersion band curvature $D^{+}=\mathrm{d}^{2} \omega / \mathrm{d} k^{2}>0$ at Fermi level intercepts. $R_{H}<0$ when the carriers have charge -e (Equation (11)).

3) By contrast, because all positive charges in $p$-Si and $A l$ are immobile and since $R_{H}>0$, the charge carriers in the magnetic field must be electrons.

4) Dispersion dynamics predicts that in crystal fields with negative dispersion $D^{-}$, the effective mass and acceleration are negative (Equation (12)).

5 ) In consequence, the velocity of electrons due to the Coulomb force $F^{e l}$ is reversed in $D^{-}$, compared with $D^{+}$.

6) In further consequence, the backward velocity reverses the otherwise conventional magnetic force $F^{m}$ and deflection (Table 1, Figure 3 ). Thus in $D^{-}$ crystal field dispersion, the Hall coefficient $R_{H}>0$.

This gives the sign of $R_{H}$ for "holey" electrons. Dispersion dynamics gives the reasons how and why those electrons behave like positive ions in the Hall effect. The argument is significant in high temperature superconductivity, where, because all positive charges are nuclear and immobile, the charge carriers can only be electrons [1] [7]. Indeed, charge pairing and reduction are common themes across the broad scope of bosonic wave particles in physics.

\section{Reduction of Wave Packets in Massive and Massless Particles}

Physically, how does it happen that a supercurrent is measured outside a superconductor, when its internal measurables, $E, B$, pair momentum $K$, pair spin $S$, resistivity $r$, etc., are all nominally zero? Reduction is mysterious when introduced as a change between time-independent wave functions: by hypothesis, the change may be represented as instantaneous at some point in space. In reality it is not so: in modern physics, resonant wave packets are the typical instruments for the force of change, and the packet is extended in both space and time. The space is divided between a direction of propagation and the corresponding transverse plane. Each of these differentiates as in Equation (1), where the group propagates with properties that are distinct from its internal transverse phase. In massive particles the group travels slower than light; the phase faster. The phase velocity $V_{p}$, in a particle with non-zero rest mass, is singular in the rest frame. Transmission of information is therefore theoretically instantaneous and Newtonian, i.e. within the transverse spatial coherence of the wave packet.

In massless photons travelling in vacuo, by contrast, the group and phase velocities share common speed $c$. In fact of course, the transmission is spread over time that is determined by the coherence $2 \sigma$, of the wave packet. This coherence determines likewise the spatial transmission, consistently with the methods of Huygen's wavelet construction and with the methods of Fresnel, Fraunhoferetc. [13]. 
Transmission of energy or momentum by massive particles, like the electron, is most easily considered in the rest frame: energy and momentum are conserved when a wave front encounters two targets simultaneously. The transverse phase velocity is singular and time is Newtonian, so that energy supplied to one target cannot be given to the other owing to released wave amplitude.

However, creation and annihilation of a massless particle, like the photon, is not so simple because $c$ is the constant velocity for both group and phase velocities. By ignoring diffraction, the transverse spatial coherence then becomes $c$ multiplied by the temporal coherence. This photon is described in Maxwell's theory by two real, sinusoidal, wave functions on mutually normal planes, one lagging in phase by $\pi / 2$. Reduction of the packet thus occurs within its spatio-temporal coherence. From Equation (2), the longitudinal coherence length is found to be $2 c \sigma$, the coherence time $2 \sigma / c$. Through $\sigma$, the coherence values depend on initial conditions for the transmitting wave packet.

What can be understood about the transverse spatial coherence? Can a wave front become wider than the coherence? Does the coherence limit beam spread? Compare the temporal coherence from section $2.1(2 \sigma / c)$ with the transverse spatial coherence from Section $2.2\left(\tan ^{-1}(\lambda / d)\right)$. It is clear that when, typically $\lambda \ll d$, the ratio $c \Delta t /(\lambda / d) \sim 2 \sigma d / \lambda$. Thus, when $\sigma>d / \lambda$, the transverse spatial coherence is determined by longitudinal temporal coherence; otherwise wave diffraction, $d / \lambda$, determines the transverse coherence. These features are in principle demonstrated by comparing diffraction of light generated by a laser source or point source. Supercurrents are massive, so we can now consider their reduction in their rest frame, i.e. in Newtonian time.

\section{Super Currents}

The solution for superconductivity was given by Bardeen, Cooper and Schrieffer [19] [20] [21]. Electrons in metallic superconductors occupy two states, normal and superconducting, i.e. at temperatures below critical $T<T_{\circ}$ and applied magnetic field $H<H_{c}$. The superconducting state consists of a pair of electrons bound together by a lattice distortion and energy gap $\Delta E$. This Cooper pair has zero net momentum $K=0$; zero net spin $S=0^{5}$; and electronic charge $2 e$. The wave is superconductive, with zero resistivity $\rho=0$ and therefore zero internal electric field $\boldsymbol{E}=0$. Meanwhile zero internal magnetic field induction $\boldsymbol{B}=0$ is demonstrated by the Meissner-Ochsenfeld effect. This is the basic state, though minor complexity arises in type II superconductors where electrons propagate quantized magnetic flux lines, and also at normal state boundaries as in the Josephson dc and ac tunneling effects. Since the normal and superconducting ${ }^{5}$ Even the intrinsic spin is problematic in dynamics. Spin is only measured in the presence of a magnetic field, so it is not obvious whether a measured magnetic moment is induced or "intrinsic" [1] [9]. Nor is it obvious that the electron is a point particle: it has magnetic moment with dimensions that include area? In particular, how can an "intrinsic spin" exist in the absence of a circulating current? Moreover, what physical transition is involved when a spin flip occurs? In dispersion dynamics, the spin is induced in non-resistive phase currents. 
states are, by supposition, time independent, anomalies naturally arise in their dynamics. In high temperature superconductors, we understand the Cooper pair to be Wannier excitons with charge $Q=0$ [1]. This is an added obstacle for conductivity. It suggests a real wave function that is Bosonic and commensurate with the lattice. How does transmission of current occur?

Consider for contrast, the more comprehensible oscillator strength in spectral transitions. This is a calculation of the relative transition rate between two stable electronic states, initial and final, excited by an operator $O$ : $\left|\left\langle y_{f}|O| y_{i}\right\rangle\right|^{2}$, typically integrated over space, time, and density of states. In a dipole transition for example, $O=e r$, where $e$ represents electronic charge. Apply such a transition to measurement of resistivity of a superconductor by the four probe technique: at $T$ $<T_{c}$ and $H<H_{c}$. When a voltage is applied between two outer terminals the supercurrent is measured at the inner sensor terminals. This current consists of normal electric charges that emerge from the positive terminal of a superconductor containing no electromagnetic fields. In dispersion dynamics this feature is not surprising: the terminals supply energy that breaks the Cooper pairs which release their normal charges to metallic terminals in Newtonian time, i.e. when considered in the rest frame. The dynamics will be governed by the transfer of thermal or electrical energy to the Cooper pairs. Each pair has zero net momentum, while any dynamic momentum that is transferred to the normal electrons at terminals, can be obtained by the pairs from the massive lattice as in X-ray diffraction and in the Mossbauer effect. Between the terminals, a supercurrent flows in absence from any internal electric or magnetic fields.

\section{Summary and Conclusion}

In dispersion dynamics, the product of the group velocity and phase velocity in a wave packet is equal to the square of the speed of light. In consequence, uncertainty is not an arbitrary limit, but a calculated expectation that has varied effects in reduction. Moreover, since immobile positive ions are not deflected by the magnetic Lorentz force, the positive Hall coefficients that are measured in certain metals and doped semiconductors must be generated by (negatively charged) "holey" electrons that exist in states having peculiar dispersive dynamics. Furthermore, in ionic high temperature superconductors, those electrons are supposedly contained in bosonic pairs of Wannier excitons. How then do supercurrents flow in the absence of internal electromagnetic fields and with zero momentum, zero spin, and zero net charge? In dispersion dynamics, the flow occurs by the reduction of time-independent waves that are consistent with packet decay, i.e. in Newtonian time between terminals. This reduction is a new solution for an unanswered problem. Following the analysis, the reduction is in principle verifiable by time-dependent measurements. Superconductivity is a physical paradigm for Boson statistics, for condensation, and for related phenomena that occur throughout the broad scope of physic. These further applications of dispersion dynamics will be objects for further study. 


\section{Conflicts of Interest}

The author declares no conflicts of interest regarding the publication of this paper.

\section{References}

[1] Bourdillon, A.J. (2017) Dispersion Dynamics in the Hall Effect and Pair Bonding in $\mathrm{HiT}_{\mathrm{T} .}$ Nova Science, Hauppauge.

[2] Bourdillon, A.J. (2012) Journal of Modern Physics, 3, 290-296. https://doi.org/10.4236/jmp.2012.33041

[3] Bourdillon, A.J. (2013) Journal of Modern Physics, 4 705-711. https://doi.org/10.4236/jmp.2013.46097

[4] Bourdillon, A.J. (2014) Journal of Modern Physics, 5, 23-28. https://doi.org/10.4236/jmp.2014.51004

[5] Bourdillon, A.J. (2015) Journal of Modern Physics, 6, 463-471. https://doi.org/10.4236/jmp.2015.64050

[6] Bourdillon, A.J. (2015) Journal of Modern Physics, 6, 2011-2020. https://doi.org/10.4236/jmp.2015.614407

[7] Bourdillon, A.J. (2017) Journal of Modern Physics, 8, 483-499. https://doi.org/10.4236/jmp.2017.84031

[8] Bourdillon, A.J. (2018) Journal of Modern Physics, 9, 1304-1316. https://doi.org/10.4236/jmp.2018.96079

[9] Bourdillon, A.J. (2018) Journal of Modern Physics, Special Issue on Magnetic Field and Magnetic Theory, 9, 2295-2307. https://doi.org/10.4236/jmp.2018.913145

[10] Aguilar-Benitez, M., Barnett, R.M., Caso, C., Conforto, G., Crawford, R.L., Cutkosky, R.E., Eichler, R.A., Eidelman, S., Groom, D.E., Hagiwara, K., Hayes, K.G., Hernandez, J.J., Hikasa, K., Höhler, G., Kawabata, S., Manley, D.M., Montanet, L., Morrison, R.J., Oliver, K.A., Porter, F.C., Roos, M., Schindler, R.H., Shrock, R.E., Stone, J., Törnqvist, N.A., Trippe, T.G., Wohl, C.G., Yost, G.P., Armstrong, B., Gieselmann, K. and Wagman, G.S. (1992) Particle Properties Data Booklet, June 1992. American Institute of Physics, from the Review of Particle Properties. Physical Review Data, D45.

[11] Pais, A. (1991) Niels Bohr's Times. Clarendon Press, Oxford, 433.

[12] Rose-Innes, A.C. and Rhoderick, E.H. (1978) Introduction to Superconductivity. 2nd Edition, Pergamon Press, Oxford.

[13] Jenkins, F.A. and White, E.W. (1937) Fundamentals of Optics. 4th Edition, McGraw-Hill, New York.

[14] Vladimirsky, Y., Bourdillon, A.J., Vladimirsky, O., Jiang, W. and Leonard, Q. (1999) Journal of Physics D: Applied Physics, 32, L114-L118. https://doi.org/10.1088/0022-3727/32/22/102

[15] Bourdillon, A.J. and Vladimirsky, Y. (2005) X-Ray Lithography on the Sweet Spot.

[16] Bourdillon, A.J. and Vladimirsky, Y. (2002) Ultra High Resolution Lithographic Imaging and Printing by Exposure near the Critical Condition. US Patent 6383697.

[17] Bourdillon, A.J. (2005) Mask Shaping in Ultra High Resolution Lithography Using Temporal and Spatial Coherence. U.S. Patent 6953643.

[18] Bourdillon, A.J. and Boothroyd, C.B. (2005) Journal of Physics D: Applied Physics, 38, 2947-2951. https://doi.org/10.1088/0022-3727/38/16/031 
[19] Cooper, L.N. (1956) Physical Review, 104, 1189-1190.

https://doi.org/10.1103/PhysRev.104.1189

[20] Bardeen, J., Cooper, L.N. and Schrieffer, J.R. (1957) Physical Review, 106, 162-164. https://doi.org/10.1103/PhysRev.106.162

[21] Bardeen, J., Cooper, L.N. and Schrieffer, J.R. (1957) Physical Review, 108, 1175. https://doi.org/10.1103/PhysRev.108.1175 